\title{
EFFICACY OF EXTRACORPOREAL SHOCKWAVE THERAPY IN MANAGEMENT CHRONIC PATELLAR TENDINOPATHY IN ATHLETES
}

Eslam M. Elkhalal* ; Ragia M. Kamel ** ;

Yasser M. Aneis ${ }^{* * *}$ and Mohamoud Abou Sayed $* * * *$

*Demonstrator of Physical Therapy of Basic Science Department, Faculty of physical therapy. Egyptian Chinese university.

** Professor Doctor of Physical Therapy of Basic Science Department, Faculty of physical therapy. Cairo University.

*** Assistant Professor Doctor of Physical Therapy of Basic Science Department, Faculty of physical therapy. Cairo University.

$* * * *$ lecturer of orthopedic and spine surgery at kasr alainy medical school, Faculty of Medicine, Cairo University.

*E-mail - eelkhalal@ecu.edu.eg

ABSTRACT

Background: There is no evidence-based, preferred treatment of choice for patellar tendinopathy. patellar tendinopathy is an overuse syndrome with pathologic changes.

Purpose: The purpose of this study is to determine the effectiveness of ESWT in management Chronic Patellar Tendinopathy in Athletes.

Materials and methods: Thirty athletes of both sexes with chronic patellar tendinopathy in the study, their age ranged from 14 to 26 years old recruited randomly. They were randomly divided into two groups (A \& B). Study group (A) (15 patients) received 3 sessions per week for 6 weeks of ice packs and isometric exercises while group (B) (15 patients) received shock wave therapy. All patients in both groups were measured using the Victorian Institute of Sport Assessment (VISA) test and a vertical jump test.

Results: There was no significant difference between the two groups pretreatment $(\mathrm{p}>0.05)$, while there was a significant increase in vertical jump height of group B compared with that of group A $(\mathrm{p}=0.001)$. There was no significant difference between the two groups pretreatment $(p>0.05)$, while there was a significant increase in VISA of group B compared with that of group A $(\mathrm{p}=0.0001)$ post treatment.

Conclusion: Extracorporeal shockwave treatment appeared to be effective and safe in the management of patients with chronic patellar tendinopathy. ESWT shoud be considered to be as a part of a treatment protocol for patellar tendinopathy.

Keywords: extracorporeal shockwave; Victorian Institute of Sport Assessment; patellar tendinopathy. 


\section{INTRODUCTION}

Patellar Tendinopathy is an overuse injury to the knee extensor mechanism caused by repeated mechanical stress from sports actions such as jumping, landing, acceleration, deceleration, and cutting. (1)

During a single exercise session, micro-tearing of the knee extensor tendons can occur as a result of the constant repetition of these movements or as a result of insufficient recovery time in between sessions. The inferior pole of the patella, where the patellar tendon inserts, is the component of the knee extensor mechanism most likely to be impacted. The insertion of the quadriceps tendon to the upper pole of the patella and the insertion of the patellar tendon into the tibial tuberosity are two less commonly affected areas of the knee. (1)

According to research, the incidence of patellar tendinopathy in elite athletes is significantly higher than in recreational athletes. Patellar tendinopathy can affect athletes of all ages, from adolescents to those in their third decade and beyond. (2)

Patellar tendinopathy shares many of the same signs and symptoms as other types of knee pathologies, such as pain when sitting for long periods of time, squatting, or climbing stairs. Typically, when performing activities that require the storage and release of energy in the patellar tendon, the amount of load-related pain increases in proportion to the demand placed on the knee extensors. When a load is applied, sudden tendon pain occurs, which usually subsides almost immediately when the load is removed. When a patient is resting, he or she is unlikely to experience pain. (1)

When it comes to jumper's knee, there is no evidence-based treatment that is preferred over the others. The condition is also characterized by a poor response to treatment, which frequently leads to the search for alternative therapies by both health professionals and patients. (1)

In the early phases of the disease, most people with jumper's knee are treated with medical and rehabilitation care. (3)

Early recognition and diagnosis of jumper's knee are critical because it can have a long and painful progression. Although non-steroidal antiinflammatory drugs (NSAIDs) have traditionally been used, they have become less judicious in recent years as more physicians have realized that the disease is not an inflammatory condition. As a result, it is possible that nonsteroidal anti-inflammatory drugs (NSAIDs) will not provide significant long-term benefit in tendinopathy. (4)

Patellar tendinopathy can be treated with Extracorporeal shock wave therapy, which is both safe and effective. Waves are generated for ESWT by accelerating a projectile through a tube at the end of which it strikes an applicator that comes into contact with the skin, using compressed air as a propellant. In order to account for this, there are significant differences between the waves generated by each technology. The first difference is that 
radial shockwaves have a more superficial effect because the maximum energy is reached at the skin's surface, as opposed to focused shockwaves, which reach a maximum energy in the focus that is located deeper within the body tissues. (5)

It has also been demonstrated that pressure waves generated by ESWT are, from a fundamental point of view, harmless. When it comes to physical therapy clinics in the Netherlands, radial shockwave therapy (ESWT) is used far more frequently (by a factor of four to one) than focused shockwave therapy (FSWT). (6)

This could be due to the fact that these devices are more reasonably priced than other options. There has not been a study to compare the effectiveness of radial shock wave in the treatment of patellar tendinopathy, so the objective of this study was to determine the effectiveness of ESWT in management Chronic Patellar Tendinopathy in Athletes.

\section{SUBJECT, MATERIALS AND METHODS}

This study was carried out on 30 athletes with patellar tendinopathy. This study was conducted in faculty of physical therapy Egyptian Chinese University. Their age from 14 to 26 year. This study was conducted from April 2020 to May 2021.

The criteria used to make the diagnosis of patellar tendinopathy were Pain at the inferior pole of the knee with training, Tenderness at the inferior pole of the patella with palpation, Absence of other knee disorders, such as patellofemoral stress syndrome, each potential study subject was screened to meet the following.

\section{Inclusion criteria:}

Age range of 14-26 years, Male and female, Diagnosis of patellar tendinopathy as described above, History of patellar tendinopathy for at least 3 months, Pain that was non-responsive to nonsteroidal anti-inflammatory medication, Athlete's basketball players and volleyball players.

\section{Exclusion criteria:}

Receiving physiotherapy (eccentric loading/stretching, etc.) within 4 weeks of randomization visit, Receiving NSAIDs or acetaminophen for any chronic condition within 1 week of randomization visit, History or physical findings of lumbar disc disease, compression syndrome, local arthrosis, or neurological abnormality, Previous surgery for patellar tendinopathy, Thrombopathy, infection, tumor, or other severe systemic disease, Receiving systemic therapeutic anticoagulation,Pregnancy,Previous ESWT treatment.

All subjects who were selected provided written informed consent before entering the study. Subjects were randomly assigned to two groups: 


\section{Group (A) Controlled group}

- Consists of fifteen patellar tendinopathy subjects received 3 sessions per Week for 6 weeks.

- Each session, subjects received Ice pack for 15 minutes and isometric exercises 20 reptations for quadriceps femoris muscle. The patient was asked to contract the muscle without lifting the heel. To check the correct contraction of the muscle, the practitioner palpates the patella to feel its proximal migration. A right knee is shown with the patient in the supine position.

\section{Group (B) Extracorporeal shockwave group}

-This group received Extracorporeal shock waves in addition to ice packs and isometric exercises.

\section{RADIAL shock wave}

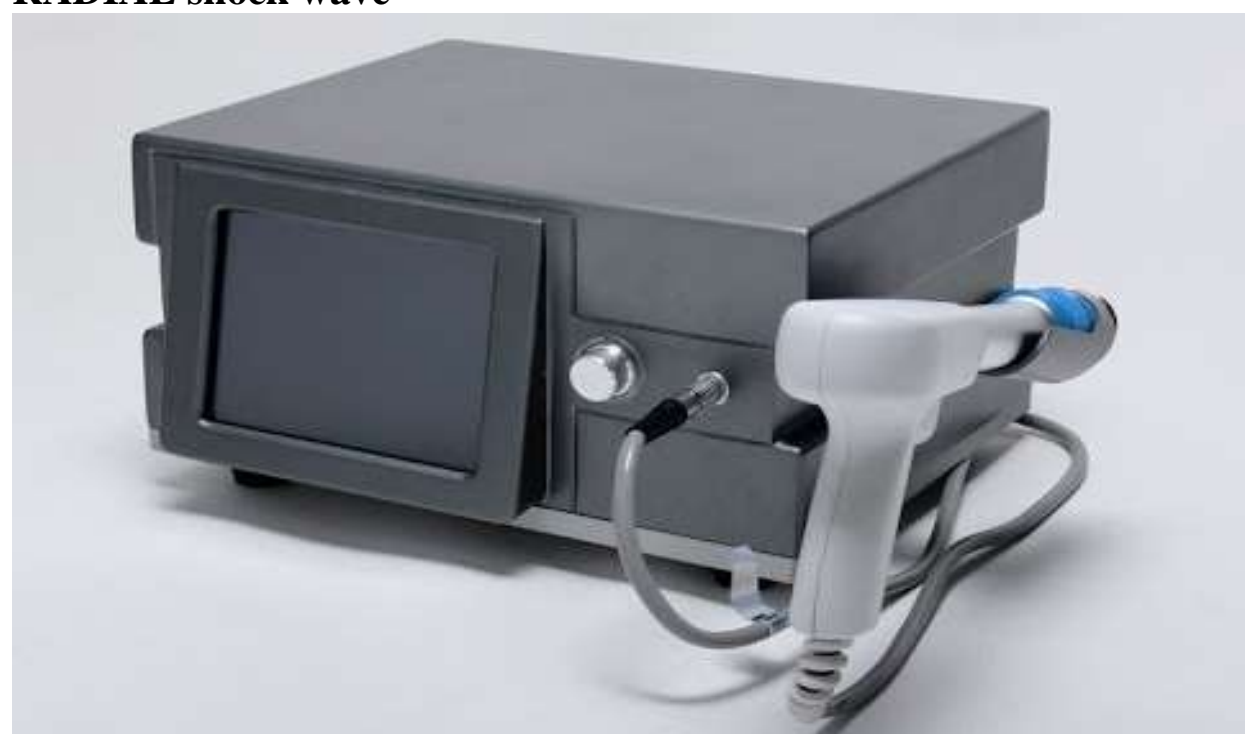

Fig.(1):

Product Name: radial shockwave therapy

Portable: portable

Application: salon, home, clinic

Theory: ultrasound

Type: shockwave therapy

Trademark: zesaybeauty

Specification: CE

Origin: China

HS Code: 90189090

Component projectile, screen

- Consists of fifteen patellar tendinopathy subjects received 3 sessions per week for 6 weeks. 
Session was performed without anesthesia using a radial shock wave device, which produces shock waves by a projectile, accelerated with a pressurized air source, which strikes a $15 \mathrm{~mm}$ diameter metal applicator. The energy produced is then transmitted to the skin as a shock wave, through a standard, commercially available ultrasound gel. The waves are dispersed radially from the application site to surrounding tissues.

Each session consists of 2000 impulses at $8 \mathrm{~Hz}$. The energy flux density is $0.12 \mathrm{~mJ} / \mathrm{mm} 2$ since this corresponds with 2.4 bar on the radial shockwave generator of the used machine.

The procedure was performed with the patient crook lying position after application of ultrasound gel on the skin overlying the distal pole of the patella and patellar tendon. The shock waves were directed from anterior to posterior. This is a dynamic process in which the area of maximal tenderness is treated circumferentially, starting from the most painful site. The average size of the treated area measured $4-8 \mathrm{~cm}$ in width and $4-8 \mathrm{~cm}$ in length, and the time of treatment ranged from 4 to 8 min.

There was a significant increase in vertical jump height and VISA post treatment compared with pretreatment which is a good indicator of increased muscle power and decreased pain in the patellar tendon.

\section{Statistical analysis}

Prior to analysis, the normality of data was checked using ShapiroWilk test. Levene's test for homogeneity of variances was conducted to test the homogeneity between groups. Data were normally distributed and there was homogeneity of variance. There were no outliers in the data as assessed boxplot. Descriptive statistics and ANOVA test was conducted for comparison of the subject characteristics between both groups. Chi squared test were conducted for comparison of sex distribution between both groups. Mixed MANOVA was conducted to compare the effect of time (pre versus post) and the effect of treatment (between groups), as well as the interaction between time and treatment on mean values of vertical jump height and VISA. The level of significance for all statistical tests was set at $p<0.05$. Statistical analysis was performed through the statistical package for social studies (SPSS) version 25 for windows.

\section{RESULTS:}

\section{Participant characteristics:}

Table 1 showed the participant characteristics of group A and B. There was no significant difference in age, weight, height, and BMI between the groups $(\mathrm{p}>0.05)$. There was no significant difference in sex distribution between groups $(\mathrm{p}=0.79)$. 
Table 1. Basic characteristics of participants.

\begin{tabular}{|l|l|l|l|}
\hline & Group A & Group B & p-value \\
\hline Age, mean \pm (SD), years & $18.2 \pm 2.04$ & $18.93 \pm 1.66$ & 0.37 \\
\hline Weight, mean \pm (SD), kg & $77.73 \pm 16.37$ & $79.13 \pm 13.51$ & 0.93 \\
\hline Height, mean \pm (SD), cm & $178.2 \pm 13.33$ & $180.53 \pm 11.6$ & 0.79 \\
\hline BMI, mean $\pm\left(\right.$ SD), kg/m ${ }^{2}$ & $24.2 \pm 2.38$ & $24.17 \pm 2.48$ & 0.97 \\
\hline Sex, n (\%) & & & \\
\hline Females & $8(53 \%)$ & $6(40 \%)$ & \multirow{2}{*}{0.76} \\
\hline Males & $7(47 \%)$ & $9(60 \%)$ & \\
\hline
\end{tabular}

SD, standard deviation; p-value, level of significance

\section{Effect of treatment on vertical jump height and VISA:}

Mixed MANOVA revealed that there was a significant interaction of treatment and time $(F=79.9, p=0.001)$. There was a significant main effect of time $(\mathrm{F}=1308.47, \mathrm{p}=0.001)$. There was a significant main effect of treatment $(\mathrm{F}=28.11, \mathrm{p}=0.001)$. Table 2 showed descriptive statistics of vertical jump height and VISA and the significant level of comparison between groups as well as significant level of comparison between before and after treatment in each group.

\section{Within group comparison}

There was a significant increase in vertical jump height and VISA post treatment compared with pretreatment in the group $\mathrm{A}$ and $\mathrm{B}(\mathrm{p}<$ $0.001)$.

\section{Between group comparison}

Between group comparison before treatment revealed a nonsignificant difference in all parameters $(\mathrm{p}>0.05)$.

Comparison between groups post treatment revealed a significant increase in vertical jump height of group B compared with that of group $\mathrm{A}(\mathrm{p}=0.001)$. (table 2$)$.

There was a significant increase in VISA of group B compared with that of group A $(\mathrm{p}=0.001)$ post treatment.

Table 2. Mean salivary flow rate and VAS-XQ at pretreatment, post I and post II of group A, B and C:

\begin{tabular}{|llll|}
\hline & \multicolumn{1}{c}{ Group A } & Group B & p-value \\
\cline { 2 - 4 } $\begin{array}{l}\text { Salivary flow } \\
\text { (ml/min) }\end{array}$ & rate & & A vs B \\
\cline { 2 - 4 } Pretreatment & $41.4 \pm 3.98$ & $41.6 \pm 2.89$ & \\
Post treatment & $43.83 \pm 4$ & $50.73 \pm 5.43$ & 1 \\
& $p=0.001$ & $p=0.001$ & 0.001 \\
VAS-XQ & & & \\
Pretreatment & $47.33 \pm 1.39$ & $48 \pm 1.51$ & \\
Post treatment & $52.1 \pm 1.6$ & $67.53 \pm 2.26$ & 0.76 \\
& $p=0.001$ & $p=0.001$ & 0.001 \\
\hline
\end{tabular}

SD, Standard deviation; $p$-value, Level of significance 


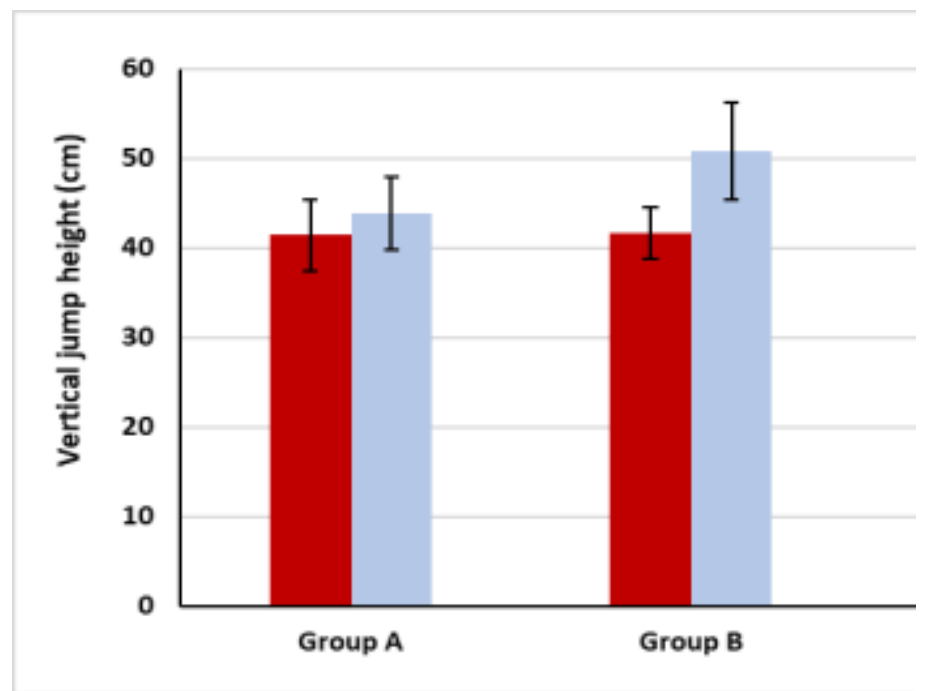

Figure (2). Mean vertical jump height pre and post treatment of group A, B.

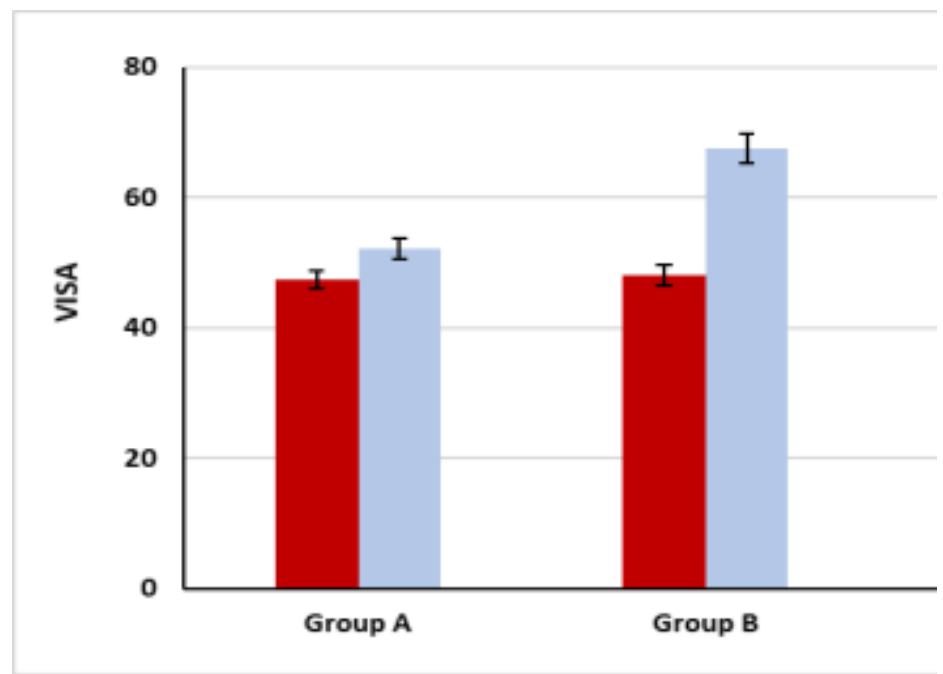

Figure (3). Mean VISA pre and post treatment of group A, B

DISCUSSION

Patellar tendinopathy is a degenerative process of the patellar tendon of unknown origin which is considered to be frequently difficult to be managed successfully, different treatment strategies have been suggested for management of patellar tendinopathy but due to the incomplete understanding of the underlying pathology, it still unclear the proper 
management of this big issue. The underlying pathology in patellar tendinopathy is considered to be a failed healing response due to a degenerative process resulting from excessive overload rather than a prostaglandin-mediated inflammatory process. (7)

In our study we used Extracorporeal Shock Wave as a treatment method for patellar tendinopathy which has a good indicator of the improvement of muscle power and reduced pain in patellar tendon which is in accordance with the study of (Pettrone F \& McCall BR 2005) who found significant differences between ESWT and placebo on pain.

(Lian et al.,2006) (7) has proposed that pain associated to patellar tendinopathy is due to Neovascularisation which is found in patellar tendinopathy, and it has been hypothesized that tendon pain is caused by sensory nerves that grow into the tendon, causing a neurogenic inflammation and pain. A further explanation of the pain in patellar tendinopathy could be the disturbance of nociceptive transmission in the nervous system by means of changed levels of substance P (SP), glutamate and tyrosine hydroxylase (TH).

The most important finding in the present study is that SWT is safe and effective treatment method, and provides significantly better results than other conservative care including the Instrument assisted Soft tissue mobilization even that the mechanism of the using of SWT in treatment of patellar tendinopathy still not fully understood, animal studies has demonstrated that ESWT may have an impact on the nociceptive transmission in the nervous system and that it can cause dysfunction of peripheral, sensory nerve fibers, resulting in pain relief as proposed by (Chung Bet al.,2002) .(8)

Also, the study of (Collins et al.,2011) (9) had found a significant difference in pain during activity in favor of the ESWT group. Which is in accordance with the finding of our study in increasing the height of jump in the Radial Shock Wave group.

ESWT is considered to be a modality for reducing pain and improving function for patients with patellar tendinopathy that might be a dose dependent effect. Based on a systematic review on the effectiveness of extracorporeal shockwave therapy in common lower limb conditions, it was concluded that ESWT-induced insignificant effects in pain scores, and patient-rated pain reduction . (10)

Also had used extracorporeal shockwave to the distal rabbit femur which had led to decrease in the concentration of substance $\mathrm{P}$ in the femoral periosteum 6 weeks after shockwave application. These studies indicated that shock wave may have selective effect on peripheral sensory unmyelinated nerve fibers without affecting large, myelinated nerve fibers. Which is going to decrees pain and preserving motor function of the muscle preserving that the muscle activity so the end result will be decreasing 
inflammation and having a good muscle power which is inconsistence with the finding of our study.

It seems that there are two different explanations of the use of SWT in the management of pain in patellar tendinopathy and there is flat contradiction to the aforementioned theory about neurovascular structures and pain. A possible explanation could be that, due to the improvement in pain and function, patients became more active, resulting in increased vascularity. In athletes the degree of neovascularization can also vary during the season but no data on sport participation were given by Wang. (11) So, in order to develop an effective ESWT treatment protocol for patellar tendinopathy it should be clear that the exact pathophysiology of patellar tendinopathy and to clarify the working mechanism of shockwaves on pathological tendon tissue and neovascularization. (12)

The findings of our study confirms that Shock Wave has improved VISA scores in the treated group significantly control group which is in agreement in the study of(Peers KH et al.,2003) (13) who conducted by Comparing outcomes of 13 patients undergoing management of patellar tendinopathy treated with surgery to those of 14 patients treated with SWT, there were significant improved VISA and VAS scores were observed in both groups at 6-month follow-up, and the period of working inability was of 6.1 weeks for the surgically treated group and 0 for the SWT group.

(Furia JP.,2006)(14) has evaluated the effects of a standardized, single treatment, low-energy protocol of radial SWT on patients with chronic patellar tendinopathy resistant to other conservative treatment and compared them with control group. (Furia JP.,2006)(14) found that the SWT-treated group reported significantly better mean of VAS and VISA scores at 1 month, 3 months, and 12 months from the treatment than the controls. Moreover, then the improvement of the VAS and VISA, patients treated with radial SWT returned to pre-injury sport activities by few months.

(Taunton et al., 2003)(15) had randomized clinical trial where the effects of ESWT for patellar tendinopathy had been evaluated on two groups. A study group of 10 received from three to five focused ESWT treatments with an energy level of $0.17 \mathrm{~mJ} / \mathrm{mm} 2$, where the control group of 10 patients had a placebo treatment. Evaluation up to 12 weeks after the last treatment included VISA score and a vertical jump test. The VISA score and vertical jump test improved significantly in the study group compared to the control group. Which is in agreement of the finding of our study in both variables (VISA score and a vertical jump test) and also it could be inferred from that ESWT should be considered important part of the rehabilitation process added to the traditional tools used in management of patellar tendinopathy.

(Bosch et al., 2009)(16) suggested another explanation for the failed treatment of ESWT in patellar tendinopathy is that athletes continued participating in their usual training program during the treatment and follow-up 
period and received no restrictions with regard to sports participation during the season.

(Zwerver et al.,2010)(17) have reached to a finding that it is possible that the total load on the tendon was too high leading to insufficient time for recovery, the authors postulated that there was a combination of collagen disorganization induced by ESWT and the mechanical load from training which might be the cause of the reduced capacity of the reparative effects of ESWT.

The optimal treatment dose has not been established as the treatment dose seems to depend on energy flex density and number of shocks. As evident by the study of (Everhart et al., 2017)(18) who observed that a significant effect of ESWT on pain reduction using energy flux density between $0.17-0.25 \mathrm{~mJ} / \mathrm{mm} 2$.

There is a considerable variability in the improvement of VISA scores from shockwave therapy which may be related to an inverse relationship between symptom improvement and total dose of treatments as suggested by (Wang et al., 2007) (11) who stated that the best results could be achieved by using an intermediate dose in a single session. While (Vetrano et al., 2013)(19) had used low to intermediate dosing over 3 weekly sessions resulting in only moderate results. which is in opposite with our findings of this study that best results of VISA scores were achieved by low to intermediate dosing over 3 weekly. A possible explanation of the difference in the finding of our study could be the age of the athletes which has a high influence of the regenerative capacity in patellar tendinopathy. also, it could be inferred that the chronicity plays an important role in the healing of the patellar tendinopathy in ESWT.

CONCLUSION \& RECOMMENDATIONS

Extracorporeal shockwave treatment appeared to effective and safe in the management of patients with chronic patellar tendinopathy. ESWT must be considered to be as a part of a treatment protocol for patellar tendinopathy.

Shockwave should be considered to be an important treatment for chronic patellar tendinopathy that have not responded to conservative measures and physical therapy. We do not recommend it for acute conditions. The best results are could be obtained when it used in combination with eccentric exercises and standardized physical therapy protocols.

\section{REFERENCES}

1- $\quad$ Santana, J.A. ; A.Mabrouk and A.L. Sherman (2020). Jumpers Knee. https://www.ncbi.nlm.nih.gov/books/NBK532969/?report=printable

2- $\quad$ Rudavsky, A. and J.Cook (2014). Physiotherapy management of patellar tendinopathy (jumper's knee). Journal of Physiotherapy, 60(3): 122-129.

3- Martens, M. ; P. Wouters ; A. Burssens and J.C. Mulier (1982): Patellar tendinitis: pathology and results of treatment. Acta orthopaedica Scandinavica. 1982 Jun [PubMed PMID: 7090767]

4- Rutland, M. ; D. O’Connell ; J.M. Brismée ; P. Sizer ; G. 
Apte and J. O'Connell (2010). Evidence-supported rehabilitation of patellar tendinopathy. North American Journal of Sports Physical Therapy : NAJSPT, 5(3): 166-178.

5- van der Worp, H. ; J. Zwerver ; M. Hamstra,; I. van den Akker- Scheek and R.L. Diercks (2014). No difference in effectiveness between focused and radial shockwave therapy for treating patellar tendinopathy: a randomized controlled trial. Knee Surgery, Sports Traumatology, Arthroscopy: Official Journal of the ESSKA, 22(9): 2026-2032.

6- Louwerens, J.K.G. and S. Gasthuis (2020). OF THE ROTATOR CUFF (Issue November)

7- Lian, Dahl J. ; P.W. Ackermann and et al.(2006). Pronociceptive and antinociceptive neuromediators in patellar tendinopathy. Am J Sports Med; 34:1801-8.

8- Chung, B. and J.P. Wiley (2002). Extracorporeal shockwave therapy. Sports Med;32:851-65.

9- Collins, E.D.H. and K.K. Jafarnia (2011) A clinical study of extractor poreal shock waves (ESW) for treatment of chronic lateral epicondylitis. Curr Orthop Pract., 22:185-192.

10- Korakakis, V.;R. Whiteley;A. Tzavara and N. Malliaropoulos (2018) The effectiveness of extracorporeal shockwave therapy in common lower limb conditions: a systematic review including quantification of patient-rated pain reduction. British Journal of Sports Medicine, 52: 387-407.

11- Wang, C.J. ; J.Y. Ko ; Y.S. Chan ; L.H. Weng and S.L. Hsu (2007): Extracorporeal shockwave for chronic patellar tendinopathy. Am J Sports Med;35:972-978

12- Malliara, P. and J. Cook (2006). Patellar tendons with normal imaging and pain: change in imaging and pain status over a volleyball season. Clin J Sport Med;16:388-91.

13- Peers, K.H. ; R.J. Lysens ; P. Brys and J. Bellemans (2003). Cross-sectional outcome analysis of athletes with chronic patellar tendinopathy treated surgically and by extracorporeal shock wave therapy. Clin J Sport Med 13:79-83.

14- Furia, J.P. (2006) High-energy extracorporeal shock wave therapy as a treatment for insertional Achilles tendinopathy. Am J Sports Med., 34:733-740.

15- Taunton, K.M. ; J.E. Taunton and K.M. Khan (2003). Treatment of patellar tendinopathy with extracorporeal shock wave therapy. BC Medical Journal; 45:500-7.

16- Bosch, G. ; M. de Mos; R. van Binsbergen ; H.T. van Schie ; C.H. van de Lest and P.R. van Weeren (2009)The effect of focused extracorporeal shock wave therapy on collagen matrix 
and gene expression in normal tendons and ligaments. Equine Vet $\mathrm{J} ;$ 41:335-41.

17- Zwerver, J. ; E. Verhagen ; F. Hartgens ; I. van den AkkerScheek and R.L. Diercks (2010). "he TOPGAME-study: efectiveness of extracorporeal shockwave therapy in jumping athletes with patellar tendinopathy. Design of a randomised controlled trial," BMC Musculoskeletal Disorders, vol. 11, article 28, 2010.

18- Everhart, J.S. ; D. Cole ; J.H. Sojka ; J.D. Higgins ; R.A. Magnussen ; L.C. Schmitt and et al. (2017):Treatment options for patellar tendinopathy: a systematic review. Arthroscopy.,33(4):861_72.

19- Vetrano, M. ; A. Castorina ; M.C. Vulpiani ; R. Baldini ; A. Pavan and A. Ferretti (2013): Platelet-rich plasma versus focused shock waves in the treatment of jumper's knee in athletes. Am J Sports Med., 41: 795-803.

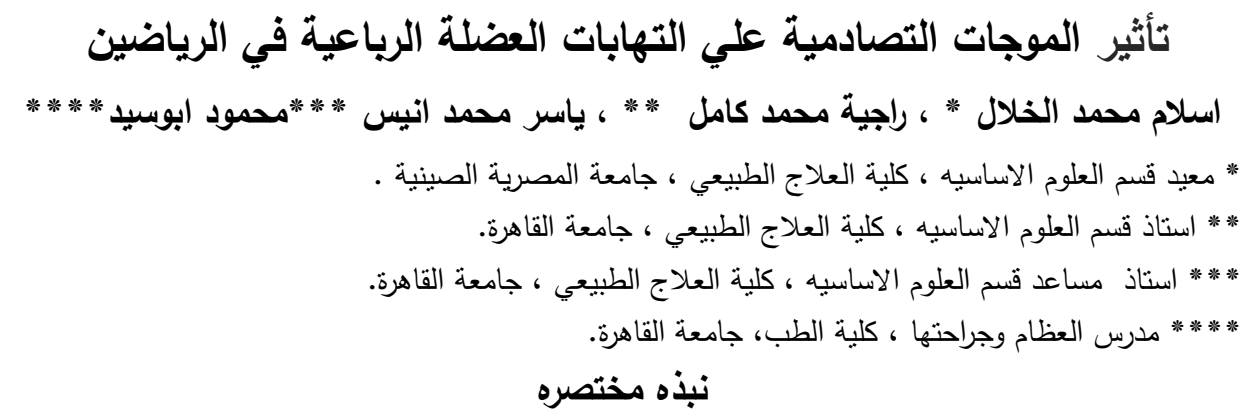

الخلقية: لا يوجد علاج مفضل قائم على الأدلة لعلاج التهابات العضلة الرباعية. التهابات العضلة الرباعية هو

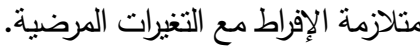
• الهذف: تحليد تاثير الموجات التصادمية علي التهابات العضلة الرباعية في الرياضبين.

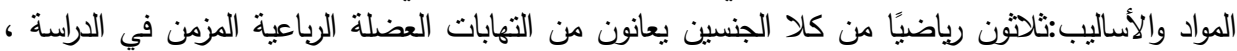

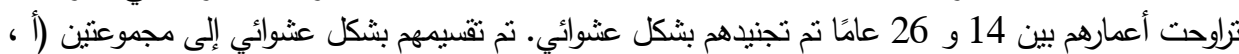

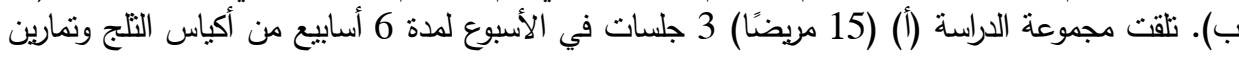

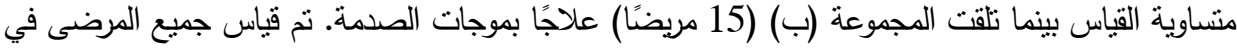

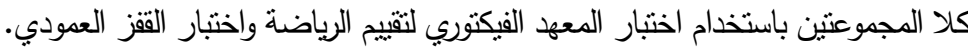

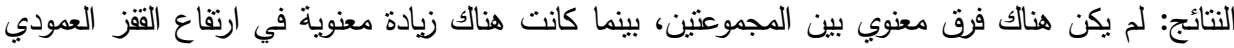

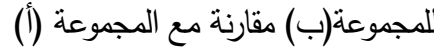

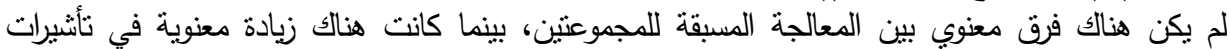
المجموعة(ب) مقارنة بالمجموعة (أ) بعد العلاج.

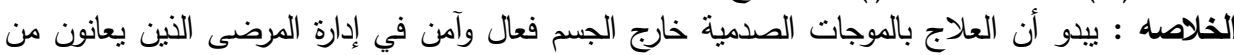

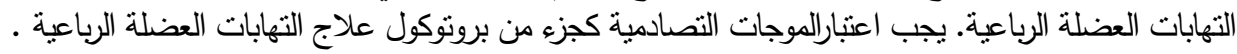

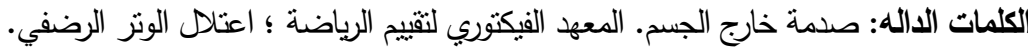

